\title{
The Axl receptor tyrosine kinase is a discriminator of macrophage function in the inflamed lung
}

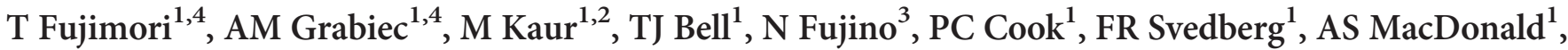 \\ RA Maciewicz ${ }^{3}$, D Singh $^{2}$ and T Hussell ${ }^{1}$
}

Much of the biology surrounding macrophage functional specificity has arisen through examining inflammation-induced polarizing signals, but this also occurs in homeostasis, requiring tissue-specific environmental triggers that influence macrophage phenotype and function. The TAM receptor family of receptor tyrosine kinases (Tyro3, AxI and MerTK) mediates the non-inflammatory removal of apoptotic cells by phagocytes through the bridging phosphatidylserine-binding molecules growth arrest-specific 6 (Gas6) or Protein S. We show that one such TAM receptor (Axl) is exclusively expressed on mouse airway macrophages, but not interstitial macrophages and other lung leukocytes, under homeostatic conditions and is constitutively ligated to Gas6. Axl expression is potently induced by granulocyte-macrophage colony-stimulating factor expressed in the healthy and inflamed airway, and by type I interferon or Toll-like receptor-3 stimulation on human and mouse macrophages, indicating potential involvement of AxI in apoptotic cell removal under inflammatory conditions. Indeed, an absence of Axl does not cause sterile inflammation in health, but leads to exaggerated lung inflammatory disease upon influenza infection. These data imply that AxI allows specific identification of airway macrophages, and that its expression is critical for macrophage functional compartmentalization in the airspaces or lung interstitium. We propose that this may be a critical feature to prevent excessive inflammation because of secondary necrosis of apoptotic cells that have not been cleared by efferocytosis.

\section{INTRODUCTION}

Tyro3, Axl, and MerTK form the TAM receptor tyrosine kinase family and bind Protein S and growth arrest-specific 6 (Gas6) proteins (Gas6 affinity for Axl $>$ Tyro3 $>$ MerTK) whose $\mathrm{N}$-terminal Gla domains bridge TAM receptors to phophatidylserine (PtdSer) on the surface of apoptotic cells, whereas the C-terminal sex hormone-binding globulin-like domain binds and activates the TAM receptor. ${ }^{1-3}$ TAM receptors are broadly expressed by cells of the vascular, nervous, reproductive, and immune systems, with mature immune cell populations predominantly expressing Axl and/or MerTK, but not Tyro3. ${ }^{2}$ There is some evidence of differential expression of TAM receptors on immune cells in health, ${ }^{4}$ and dynamic regulation of Axl and MerTK expression on macrophages by pro- and anti-inflammatory factors has recently been characterized. ${ }^{5}$ In the innate immune system, TAM receptors inhibit inflammation during apoptotic cell efferocytosis via a negative feedback loop involving activation of suppressor of cytokine signaling-1 and -3 that inhibit cytokine and Toll-like receptor (TLR) signaling pathways. ${ }^{6-8}$ An absence of TAM receptors results in impaired apoptotic cell clearance, the generation of antibodies to self-cellular antigens, ${ }^{7,9}$ and heightened responses to endotoxin ${ }^{9,10}$ and inflammatory cytokines. ${ }^{11}$

Given a critical role of TAM receptors in suppressing immune responses, it is not surprising that defects in the TAM receptor system have been identified in patients with autoimmune diseases, including multiple sclerosis and systemic lupus erythematosus. ${ }^{7,12}$ However, although inhibition of innate inflammation is essential to prevent autoimmunity during apoptotic cell clearance, prolonged engagement of TAM receptors may cause a state of unresponsiveness in antigenpresenting cells required to clear pathogenic microorganisms.

\footnotetext{
${ }^{1}$ Manchester Collaborative Centre for Inflammation Research, Manchester University, Core Technology Facility, Manchester, UK. ${ }^{2}$ University of Manchester NIHR Translational Research Facility, Manchester Academic Health Science Centre, University Hospital of South Manchester Foundation Trust, Manchester, UK and ${ }^{3}$ AstraZeneca R\&D Mölndal, Mölndal, Sweden. Correspondence: T Hussell (tracy.hussell@manchester.ac.uk)

${ }^{4}$ These authors contributed equally to this work.
} 
It is noteworthy that elevated Gas6 plasma levels are observed in patients with severe sepsis ${ }^{13}$ and MerTK is elevated on monocytes from patients with septic shock compared with trauma patients and healthy controls, and is linked to an adverse outcome. ${ }^{14}$

The mucosal immune system of the lung requires a fine balance between the ability to mount adequate responses to pathogens entering the respiratory tract, and the control of inflammatory processes that might arise from accumulation of large quantities of structural and infiltrating immune cells that undergo apoptosis. ${ }^{15}$ Despite this critical regulatory role of efferocytosis in the lung mucosa, little is known about TAM receptors or their ligands in the healthy or inflamed lung. Moreover, in light of recent evidence of an oncogenic function of TAM receptors and initial attempts to block TAM receptor signaling in cancer patients, ${ }^{16}$ it is of great interest to understand how manipulation of TAM receptor signaling could affect susceptibility to lung infections. We now show that, unlike the reported dominance of MerTK on murine primary macrophages ${ }^{4}$ and the human macrophage cell line U937, ${ }^{6,17}$ Axl was specifically and constitutively expressed on airway, but not interstitial lung macrophages. Inhalation of influenza virus or stimulation of macrophages in vitro with viral pathogenassociated molecular patterns or type I interferon (IFN) specifically upregulated Axl. Finally, we show that $\mathrm{Axl}^{-1-}$ mice were unable to resolve influenza-induced inflammation causing an accumulation of apoptotic cells and necrotic cell debris. This study provides clear evidence for a constitutive and critical role for the TAM receptor Axl in lung immune homeostasis and in resolution of viral inflammatory lung disease.

\section{RESULTS}

The TAM receptor AxI is exclusively expressed on airway macrophages in the homeostatic lung

Murine airway macrophages in homeostasis were characterized as $\mathrm{CD} 11 b^{\text {lo }} \mathrm{CD} 11 \mathrm{c}^{\mathrm{hi}} \mathrm{F} 4 / 80^{+} \mathrm{Ly}_{6 \mathrm{G}}^{-}$, were $95 \%$ pure in health (Figure 1a), and expressed high levels of Axl and MerTK, but not Tyro3 (Figure 1b). Airway lavage does not remove all airway macrophages, which can be observed in dissociated lung interstitial tissue. Here, also present were monocyte/macrophages that were $\mathrm{CD} 11 \mathrm{~b}^{\text {hi }} \mathrm{CD} 11 \mathrm{c}^{\text {intermediate }}$ and monocytes that were $\mathrm{CD} 11 \mathrm{~b}^{\text {hi }} \mathrm{CD} 11 \mathrm{c}^{\text {lo }}$ (Figure 1c). Axl and MerTK were almost exclusively expressed by $\mathrm{CD} 11 \mathrm{~b}^{\mathrm{lo}} \mathrm{CD} 11 \mathrm{c}^{\mathrm{hi}}$ airway macrophages at this site, while we did not detect significant levels of Tyro3 on any of the analyzed populations (Figure 1d). High Axl protein expression was confirmed by western blot analysis in purified airway macrophages from wild type but not $\mathrm{Axl}^{-1-}$ mice (Figure 1e). The majority of airway macrophages co-expressed both TAM receptors (Figure 1f). Interestingly, airway macrophages were the only immune cell population of the lung expressing high levels of Axl: we failed to detect Axl protein on neutrophils, eosinophils, T cells, NK cells, and only a very low level of Axl was detected on dendritic cells residing in the lung under homeostatic conditions (Supplementary Figure S1 online).
We next compared airway macrophage TAM receptor expression with macrophages in different anatomical locations. Airway macrophages expressed $\sim 20$-fold higher levels of Axl mRNA compared with peritoneal macrophages (Figure 2a), whereas expression of MerTK mRNA was more evenly distributed between these macrophage populations (Figure $\mathbf{2 b}$ ). Consistently, within the analyzed macrophage populations, Axl protein expression at homeostasis was restricted to mucosal macrophages in the intestinal tract and airway, with the most dominant expression on airway macrophages (Figure 2c), whereas MerTK was more widely expressed (Figure 2d), indicating a specific role for $\mathrm{Axl}$ in apoptotic cell clearance from the airways.

Specific expression of Axl on airway macrophages may reflect constituents of the healthy lung microenvironment. This hypothesis is supported by the exclusive ability of granulocytemacrophage colony-stimulating factor (GM-CSF), but not macrophage colony-stimulating factor (M-CSF), to induce Axl mRNA (Figure 3a) and protein (Figure 3c) expression in the course of differentiation of bone marrow-derived macrophages (BMDMs), an influence clearly visible also by flow cytometry (Figure 3d). High levels of MerTK expression, however, were detected in BMDMs differentiated by either M-CSF or GMCSF (Figure $\mathbf{3 b}$ and $\mathbf{e}$ ). Furthermore, Axl expression could also be selectively induced by GM-CSF, but not by M-CSF, on otherwise Axl-negative terminally differentiated macrophages from the murine peritoneal cavity (Figure $\mathbf{3 f}$ and $\mathbf{g}$ ). Given a critical role of GM-CSF in airway macrophage development, ${ }^{18,19}$ this observation indicates that GM-CSF might act as a dominant signal for macrophage expression of Axl in homeostasis.

\section{The TAM receptor ligand Gas 6 is constitutively bound to $\mathbf{A x l}$}

TAM receptors recognize externalized PtdSer on apoptotic cells via the bridging ligands Gas6 or protein S. ${ }^{2}$ All of the airway macrophages expressing Axl also co-stained with Gas6 by flow cytometry, suggesting constitutive binding in the steady state (Figure 4a). This was confirmed in $\mathrm{Axl}^{-1-}$ mice where both Axl (Figure 4b) and Gas6 (Figure 4c) expression was lost. The observation that Gas6 is not detected on $\mathrm{Axl}^{-/-}$airway macrophages despite high MerTK expression on these cells (Figure 1b) also indicated that constitutive binding of Gas6 in the absence of apoptotic cells is restricted to Axl. We next examined whether macrophage populations can also act as a source of Gas6 for Axl-expressing cells by profiling Gas6 mRNA expression. Low levels of Gas6 mRNA were observed in peritoneal or airway macrophages $\left(\mathrm{CD} 11 b^{\text {lo }} \mathrm{CD} 11 c^{\text {hi }}\right)$, whereas elevated Gas6 expression was observed in lung monocytemacrophages $\left(\mathrm{CD} 11 \mathrm{~b}^{\text {hi }} \mathrm{CD} 11 \mathrm{c}^{\text {lo }}\right.$; Figure 4d). This suggests that macrophages driven by particular stimuli can produce Gas6, whereas others can bind it in a paracrine manner. To test this we derived BMDMs using M-CSF or GM-CSF; importantly, only the former expressed substantial levels of Gas6 mRNA (Figure 4e). Upon incubation with exogenous Gas6, only GM-CSF-differentiated BMDMs, which express high levels of 


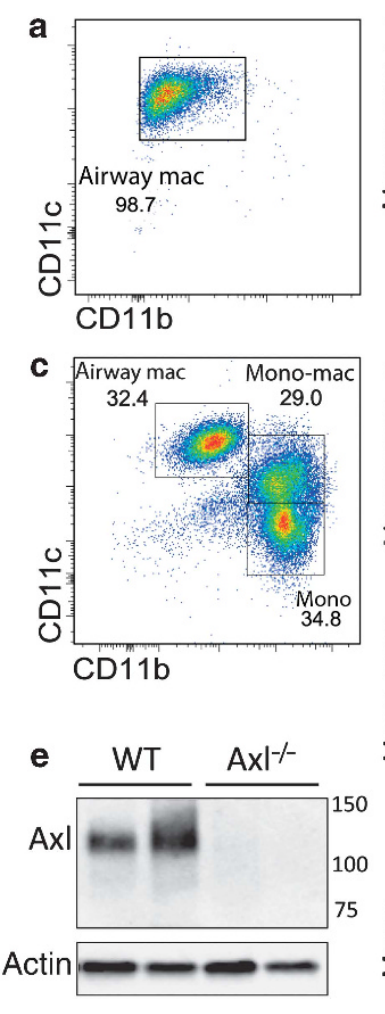

$\mathbf{f}$

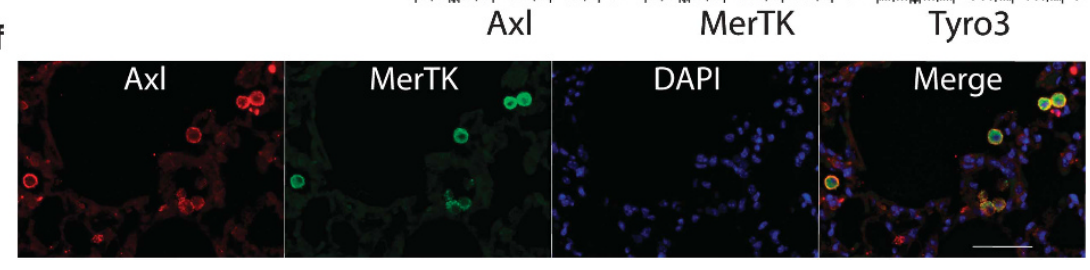

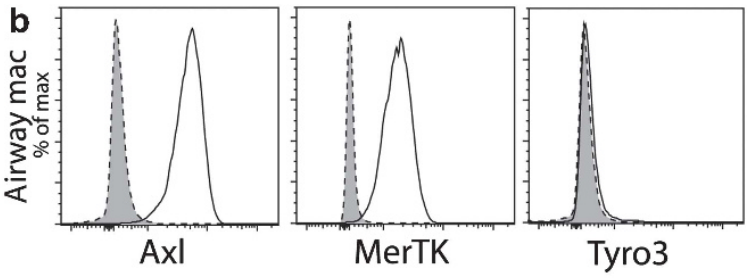
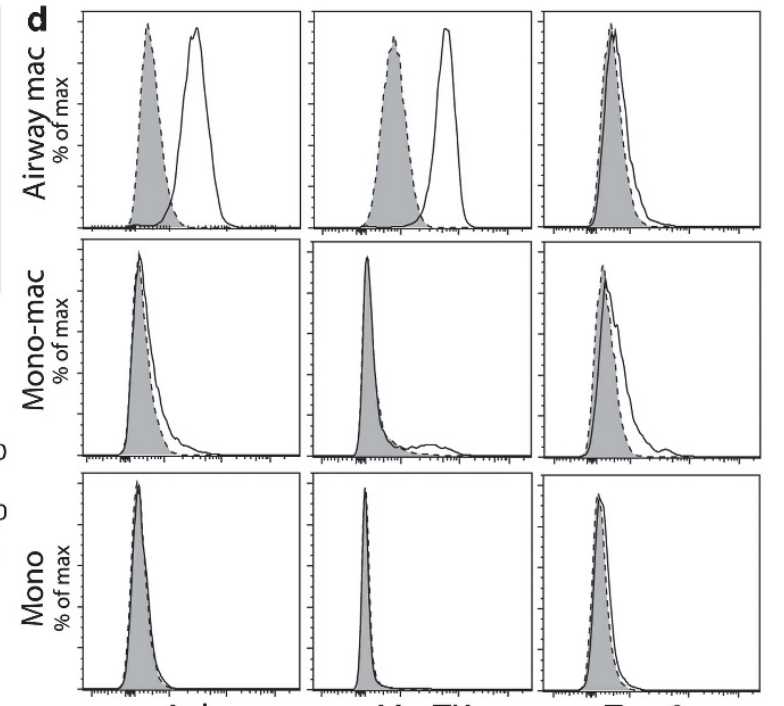

Axl

Figure $1 \mathrm{Axl}$ is primarily expressed on airway macrophages in homeostasis. Flow cytometric analysis of F4/80-positive cells in (a) airway and (c) dissociated interstitial lung tissue by $\mathrm{CD} 11 \mathrm{~b}$ and $\mathrm{CD} 11 \mathrm{c}$ expression. Airway macrophages (airway mac) are defined as CD $11 \mathrm{~b}^{\mathrm{lo}} \mathrm{CD} 11 \mathrm{c}^{\mathrm{hi}} ;$; lung monocytemacrophages (mono-mac) are defined as CD11 b ${ }^{\text {hi }} C D 11 c^{\text {intermediate; }}$ lung monocytes (mono) are defined as CD $11 b^{\text {hi }} C D 11 c^{\text {lo }}$. (b and d) Flow cytometric analysis of Axl, MerTK, and Tyro3 expression on airway macrophages, lung monocyte-macrophages, and lung monocytes. Specific staining, solid line/open. Isotype control, dotted line/shaded. (e) Western blot analysis of Axl protein expression in airway macrophages isolated from wild-type (WT) or $\mathrm{Axl}^{-1}$ - mice. Each lane represents lysates from individual mice. (f) Paraffin-embedded lung sections from naïve mice stained with antibodies specific for Axl (red) or MerTK (green), and counter-stained with 4',6-diamidino-2-phenylindole (DAPI; blue). Bar $=50 \mu \mathrm{m}$. Data are representative of two independent experiments with four to five mice (all except f) or two independent experiments with two mice per group (f, immunohistochemistry).

Axl (Figure 4f), acquired Gas6 signal on the cell surface (Figure 4g). On the other hand, neither BMDMs from $\mathrm{Axl}^{-1-}$ mice nor Axl-negative M-CSF-differentiated BMDMs from WT mice were able to bind exogenous Gas6 (Figure 4g), confirming the specificity of homeostatic Axl-Gas6 interaction.

\section{Viral triggers and influenza infection induce Axl expression on macrophages}

Macrophage populations in the airspaces and lung change dramatically during influenza infection; in wild-type mice intranasal influenza $\mathrm{H} 1 \mathrm{~N} 1$ infection induces a viral titerdependent weight loss that correlates with the extent of the inflammatory cell infiltrate. ${ }^{20,21}$ We used a dose that causes up to $25 \%$ weight loss with recovery (Figure 5a). Infection with influenza resulted in monocyte/macrophages recruitment to the airspaces leading to the presence of three main populations (Figure 5b). As in health (Figure 1b), all resident airway macrophages $\left(\mathrm{CD} 11 \mathrm{~b}^{\mathrm{lo}} \mathrm{CD} 11 \mathrm{c}^{\mathrm{hi}}\right)$ were Axl- and MerTKpositive (Figure 5c). In contrast to uninfected animals (Figure 1d), following viral infection infiltrating monocyte/ macrophages $\left(\mathrm{CD} 11 \mathrm{~b}^{\text {hi }} \mathrm{CD} 11 \mathrm{c}^{\text {intermediate }}\right)$ also expressed Axl and MerTK, albeit to a lower intensity than airway macrophages (Figure 5d). No expression of Axl or MerTK was observed on infiltrating monocytes (Figure 5e). The number of Axl-expressing macrophages and the levels of soluble Axl in airway lavage fluid (Figure $\mathbf{5 f}$ and $\mathbf{g}$ ) increased with infection, peaking at the point of maximal weight loss and cellular infiltrate, then returning to a level above that observed pre-infection. Monocyte/macrophages infiltrating the inflamed airway can therefore increase Axl expression during the course of viral infection. 


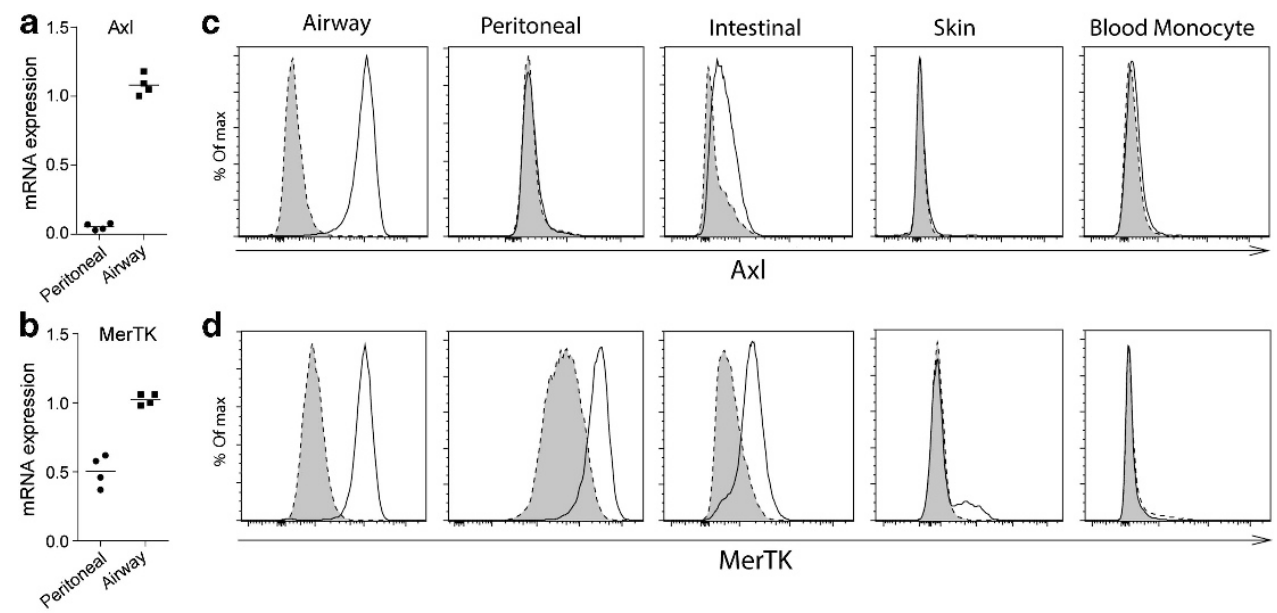

Figure 2 Axl expression at homeostasis is restricted to mucosal macrophages. Axl (a) and MerTK (b) relative mRNA expression in purified airway and peritoneal macrophages. Symbols represent data from individual mice and lines represent the mean value. Flow cytometric analysis of (c) Axl and (d) MerTK expression on airway, peritoneal, intestinal and ear skin macrophages, and blood monocytes. Specific staining, solid line/open. Isotype control, dotted line/shaded. (c and d) are representative of two independent experiments with five mice, except for the ear skin data; one experiment with five mice.
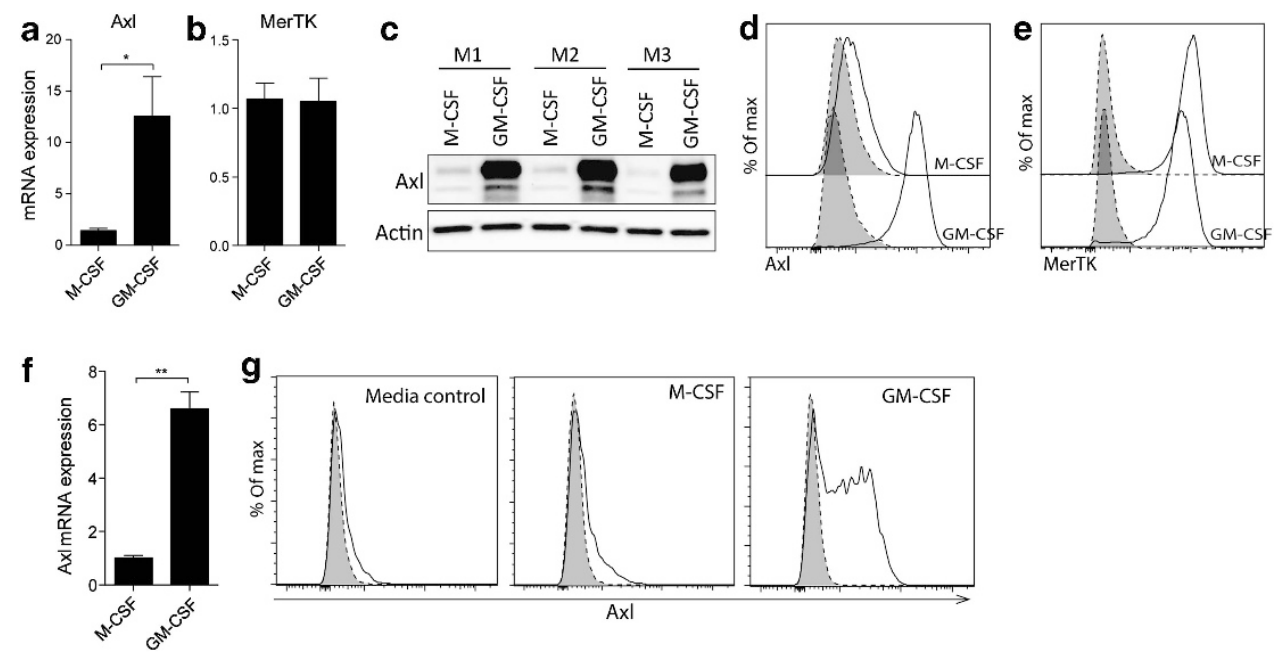

Figure 3 GM-CSF drives Axl expression on macrophages. (a) Axl and (b) MerTK relative mRNA expression in M-CSF- or GM-CSF-differentiated bone marrow-derived macrophages (BMDMs). (c) Western blot analysis of Axl protein expression in M-CSF- or GM-CSF-differentiated BMDMs. M1-M3 represent lysates of cells obtained from individual mice. Expression of (d) Axl and (e) MerTK in M-CSF- or GM-CSF-differentiated BMDMs analyzed by flow cytometry. Specific staining, solid line/open. Isotype control, dotted line/shaded. (f) Relative mRNA expression of Axl in peritoneal macrophages measured after either M-CSF $\left(50 \mathrm{ng} \mathrm{ml}^{-1}\right)$ or GM-CSF $\left(50 \mathrm{ng} \mathrm{ml}^{-1}\right)$ stimulation for $24 \mathrm{~h}$. (g) Flow cytometric analysis of regulation of Axl expression on peritoneal macrophages isolated from naïve mice. Peritoneal macrophages were left untreated or were stimulated for $48 \mathrm{~h}$ with $\mathrm{M}-\mathrm{CSF}\left(50 \mathrm{ng} \mathrm{ml}^{-1}\right)$ or GM-CSF $\left(50 \mathrm{ng} \mathrm{ml}^{-1}\right)$. Specific staining, solid line/open. Isotype control, dotted line/shaded. Data are representative of two independent experiments with three or four mice. Quantitative PCR data are expressed as the mean relative gene expression \pm s.e.m. of three or four individual mice (a, $\mathbf{b}$, and $\mathbf{f}$ ). ${ }^{\star} P<0.05$, ${ }^{\star \star} P<0.01$ vs. corresponding group; unpaired $t$-test.

To assess the virally induced factors that might influence macrophage expression of Axl, we stimulated peritoneal macrophages or M-CSF-differentiated BMDMs in vitro with the viral TLR3 agonist polyinosinic/polycytidylic acid (p(I:C)) or with IFN- $\alpha$. Both of these stimuli induced Axl mRNA (Figure 6a and $\mathbf{c}$ ) and protein expression (Figure $\mathbf{6 b}$ and $\mathbf{d}$ ), demonstrating that both endogenous and exogenous triggers associated with viral infection drive Axl expression. This regulatory mechanism was conserved between mouse and human cells, as p(I:C) also upregulated Axl mRNA (Figure 6e) and protein expression (Figure 6f) in otherwise Axl-negative human monocyte-derived macrophages, as did IFN- $\alpha$ (Figure 6g). Interestingly, $\mathrm{p}(\mathrm{I}: \mathrm{C})$ also upregulated $\mathrm{Axl}$ on human airway macrophages (Figure 6h). As p(I:C) mimics the component of viral genome, stimulation of macrophages with $\mathrm{p}(\mathrm{I}: \mathrm{C})$ triggers IFN- $\alpha$ production. To determine if $\mathrm{p}(\mathrm{I}: \mathrm{C})$-mediated $\mathrm{Axl}$ 

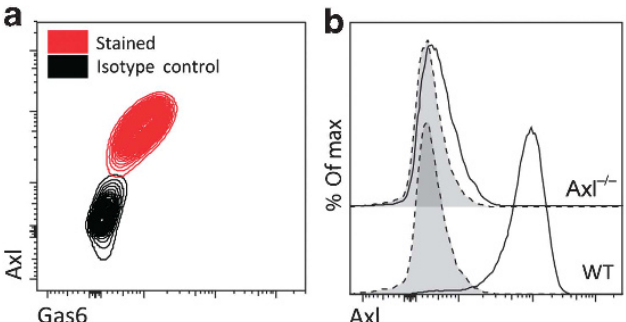

Axl

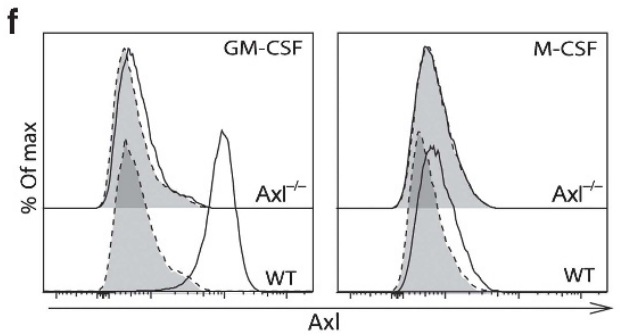

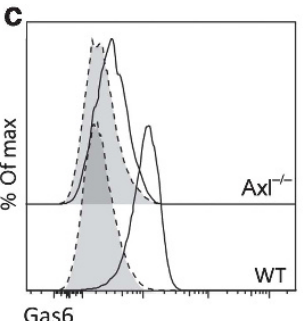
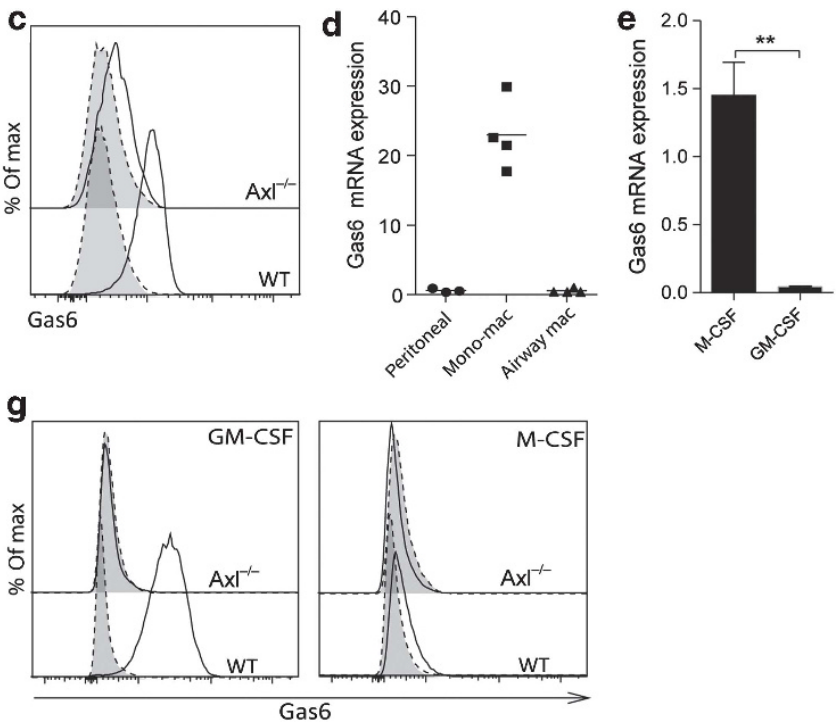

Figure 4 Constitutive binding of Gas 6 to the surface of airway macrophages through specific interaction with Axl. (a) Flow cytometric analysis of Axl and Gas6 co-expression on airway macrophages. Flow cytometric analysis of (b) Axl and (c) Gas6 expression on airway macrophages isolated from wild-type (WT) or Axl ${ }^{-1-}$ mice. (d) Relative mRNA expression of Gas6 in purified peritoneal macrophages, lung monocyte-macrophages (mono-mac), and airway macrophages. Symbols represent data from individual mice and lines represent the mean value. (e) Relative mRNA expression of Gas6 in M-CSF- or GM-CSF-differentiated BMDMs. (f and $\mathbf{g}$ ) Analysis of Axl-dependent binding of Gas6 to M-CSF- or GM-CSF-differentiated BMDMs isolated from WT or $\mathrm{AxI}^{-/-}$mice. BMDMs were left untreated or were incubated with recombinant Gas 6 for $1 \mathrm{~h}$ and then surface levels of (f) Axl and (g) Gas 6 were analyzed by flow cytometry. (f) Specific staining, solid line/open. Isotype control, dotted line/shaded. (g) + Gas6, solid line/open. - Gas6, dotted line/shaded. Data are representative of at least two independent experiments with four mice. ${ }^{\star \star} P<0.01 \mathrm{vs}$. corresponding group; unpaired $t$-test.
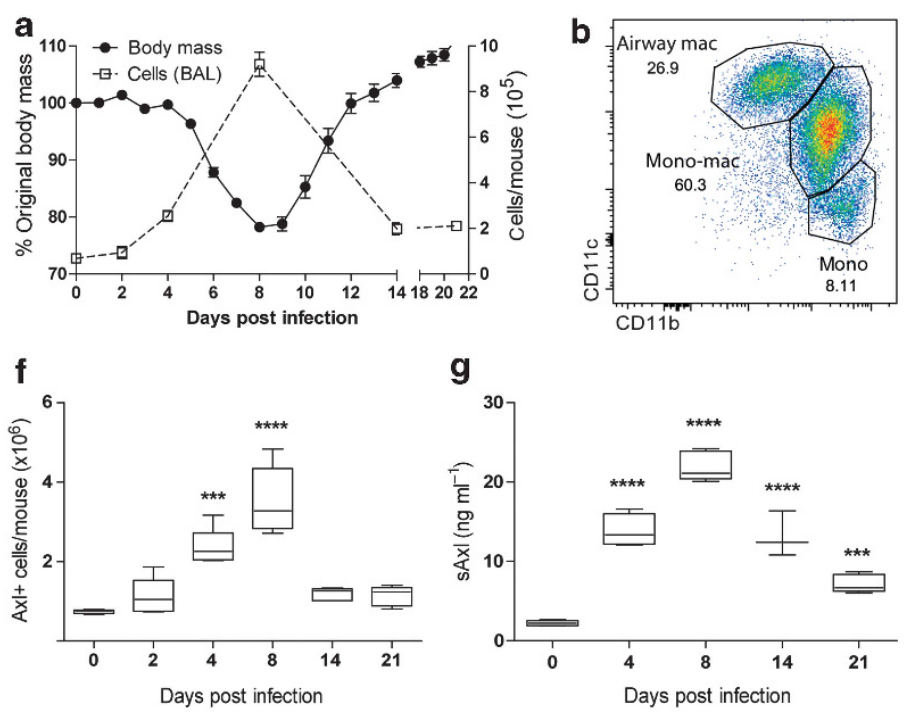
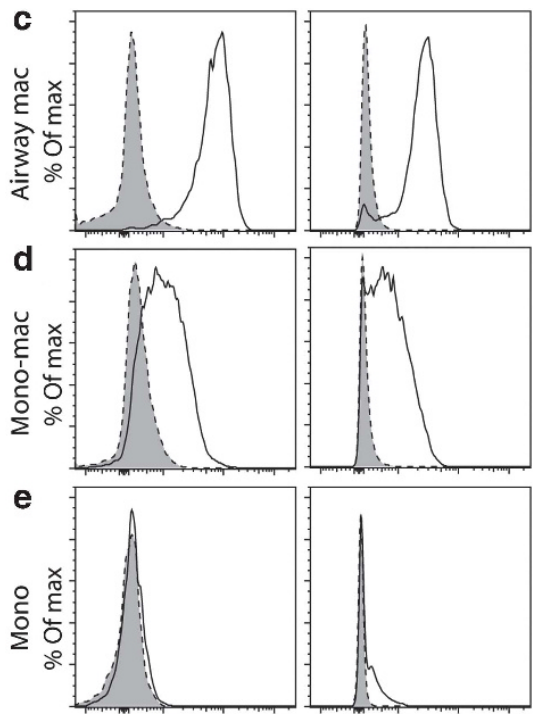

Axl

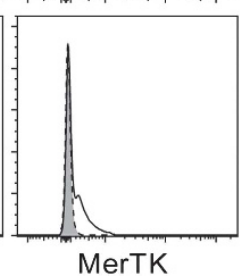

Figure 5 Increase in the numbers of Axl-expressing cells during influenza. (a) Change in body mass of wild-type mice infected with 7.5 p.f.u. influenza (closed symbol) and change in total cell numbers in the bronchoairway lavage (BAL; open symbol), assessed at 0-21 days after infection. (b) Flow cytometric analysis of F4/80-positive cells in the BAL from influenza-infected mice (8 days post infection) by CD11b and CD11c expression. Airway macrophages (airway mac), lung monocyte-macrophages (mono-mac), and lung monocytes (mono) are defined as in Figure 1a. (c-e) Flow cytometric analysis of Axl and MerTK expression on airway macrophages, monocyte-macrophages, and monocytes in the BAL from mice infected with influenza (8 days post infection). Specific staining, solid line/open. Isotype control, dotted line/shaded. (f) Number of Axl-expressing airway macrophages and monocyte-macrophage populations in the total lung during the course of influenza infection (0-21 days post infection). (g) Amount of soluble Axl (sAxl) released into the BAL fluid during the course of influenza infection. Data are representative of two independent experiments with five mice. ${ }^{* \star \star} P<0.001$, ${ }^{* * * \star} P<0.0001$ vs. naïve group (day 0 ); one-way analysis of variance.

upregulation is directly dependent on the release of IFN- $\alpha$, we treated human monocyte-derived macrophages with an antitype I IFN receptor (IFNAR) neutralizing antibody before
$p(I: C)$ stimulation and subsequently assessed the expression of Axl. Treating human monocyte-derived macrophages with anti-IFNAR antibody blocked the induction of Axl protein 


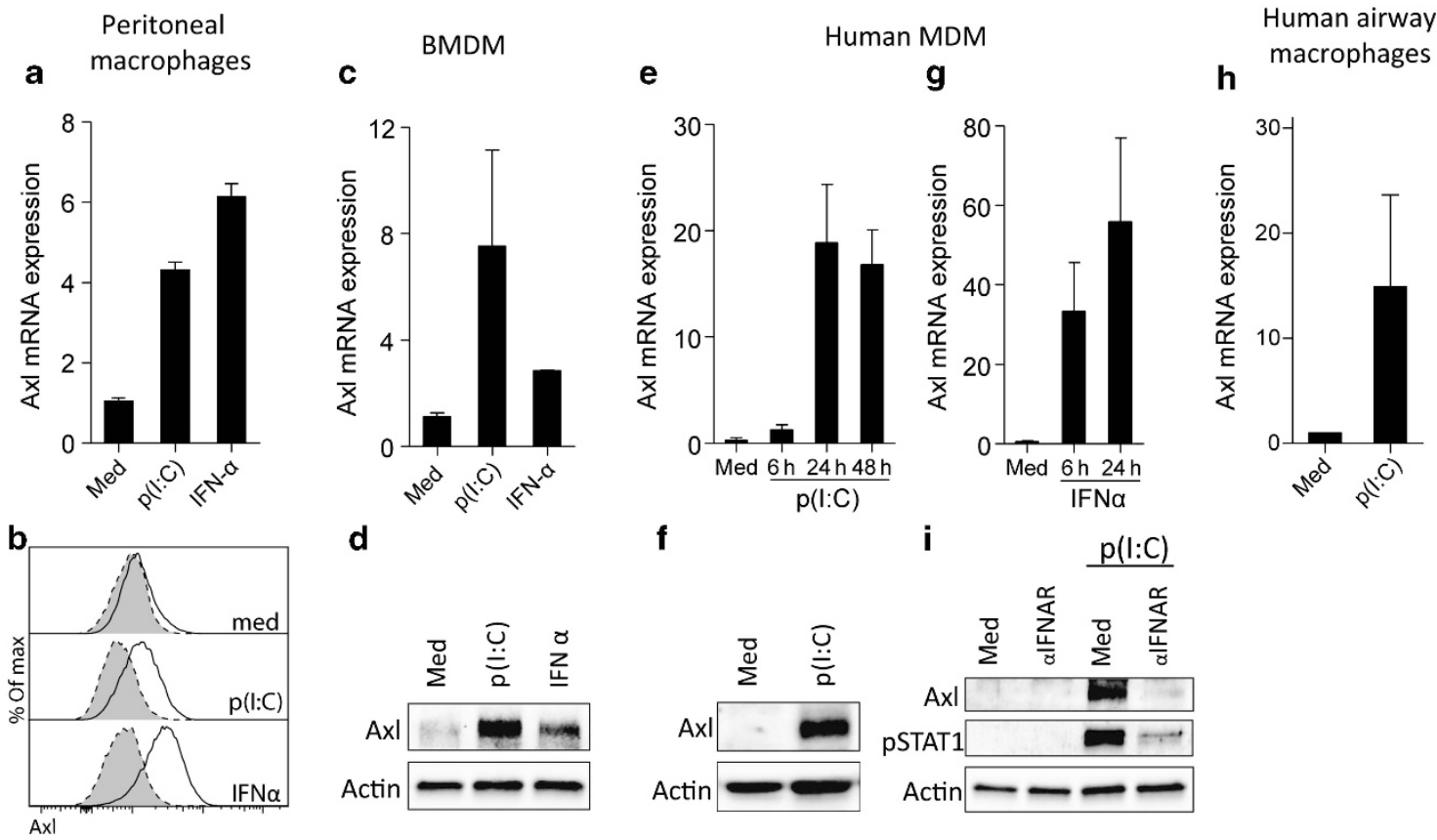

Figure 6 Axl induction is triggered by immune responses to viruses. (a) Relative mRNA expression of Axl in peritoneal macrophages isolated from naïve mice measured after $24 \mathrm{~h}$ stimulation with $\mathrm{p}(\mathrm{I}: \mathrm{C})\left(10 \mu \mathrm{g} \mathrm{ml}^{-1}\right)$ or IFN- $\alpha\left(100 \mathrm{ng} \mathrm{ml}^{-1}\right)$. (b) Flow cytometric analysis of Axl expression on peritoneal macrophages stimulated for $48 \mathrm{~h}$ with $\mathrm{p}(\mathrm{I}: \mathrm{C})\left(10 \mu \mathrm{g} \mathrm{ml}^{-1}\right)$ or IFN- $\alpha\left(100 \mathrm{ng} \mathrm{ml}^{-1}\right)$. Specific staining, solid line/open. Isotype control, dotted line/shaded. (c) Relative mRNA expression of Axl and (d) Western blot analysis of Axl protein expression in M-CSF-differentiated bone marrow-derived macrophages (BMDMs) stimulated with $\mathrm{p}(\mathrm{I}: \mathrm{C})\left(10 \mu \mathrm{g} \mathrm{ml}^{-1}\right)$ or IFN- $\alpha\left(100 \mathrm{ng} \mathrm{ml}^{-1}\right)$ for $24 \mathrm{~h}$. (e) Relative mRNA expression of Axl in human monocyte-derived macrophages (MDMs) measured 6, 24, and $48 \mathrm{~h}$ after $\mathrm{p}(\mathrm{l}: \mathrm{C})\left(50 \mu \mathrm{g} \mathrm{ml}^{-1}\right)$ stimulation. (f) Western blot analysis of Axl protein expression in human MDMs stimulated with $\mathrm{p}(\mathrm{l}: \mathrm{C})\left(50 \mu \mathrm{g} \mathrm{ml}^{-1}\right)$ for $48 \mathrm{~h}$. (g) Relative mRNA expression of Axl in human MDMs after 6 and $24 \mathrm{~h}$ stimulation with IFN- $\alpha\left(1,000 \mathrm{U}\right.$ ml $\left.{ }^{-1}\right)$. (h) Relative mRNA expression of Axl in human airway macrophages stimulated with $\mathrm{p}(\mathrm{l}: \mathrm{C})\left(1 \mu \mathrm{g} \mathrm{ml}^{-1}\right)$ for $4 \mathrm{~h}$. (i) Western blot analysis of Axl protein expression and STAT1 phosphorylation in human MDMs stimulated with $\mathrm{p}(\mathrm{I}: \mathrm{C})\left(50 \mu \mathrm{gl}^{-1}\right)$ for $48 \mathrm{~h}$ in the absence or presence of an anti-IFNARneutralizing antibody $\left(5 \mathrm{\mu g} \mathrm{ml}^{-1}\right)$. Quantitative PCR data are expressed as the mean relative Axl expression \pm s.e.m. of three or four individual mice (a,c) or donors $(\mathbf{e}, \mathbf{g}, \mathbf{h})$. Protein expression data are representative of three or four mice $(\mathbf{b}, \mathbf{d})$ or donors $(\mathbf{f}, \mathbf{i})$.

expression by $\mathrm{p}(\mathrm{I}: \mathrm{C})$ in a STAT1 phosphorylation-dependent manner (Figure 6i), showing that Axl upregulation by $\mathrm{p}(\mathrm{I}: \mathrm{C})$ is dependent on IFN- $\alpha$ release.

\section{Axl is required for resolution of lung inflammatory disease upon influenza infection}

$\mathrm{Axl}^{-1-}$ mice did not show any alterations in lung immune cell composition in homeostasis (Supplementary Figure S2), suggesting that the presence of MerTK is sufficient for the clearance of apoptotic cells under homeostatic conditions. However, in light of the high Axl expression on airway macrophages, but not other lung leukocytes, and rapid increases in the numbers of Axl-positive cells in the airways during influenza infection, we hypothesized that Axl has a distinctive, but complementary, role to MerTK during inflammatory lung disease. Indeed, upon influenza infection $\mathrm{Axl}^{-1-}$ mice displayed enhanced weight loss, with impaired recovery, requiring the experiment to be terminated (Figure 7a). Exacerbated disease was associated with elevated inflammatory cytokine/chemokine release into the airways (Figure $7 \mathbf{b}$ and $\mathbf{c}$ ). The number of total cells in the airway was also increased in the absence of Axl (Figure 7d), mostly accounted for by increases in neutrophils and $\mathrm{CD} 4^{+}$and $\mathrm{CD} 8{ }^{+} \mathrm{T}$ cells (Figure $7 \mathbf{e}-\mathbf{g}$ ). Enhanced severity of influenza infection in mice lacking Axl was not due to a delay in viral clearance (Figure $\mathbf{7 h}$ ) and is likely a result of secondary necrosis of unefferocytosed apoptotic cells. Indeed, the numbers of early and late apoptotic cells (Figure $7 \mathbf{i}$ and $\mathbf{j}$ ), as well as nucleosome release (Figure $7 \mathbf{k}$ )-indicative of necrosis or secondary necrosis of apoptotic cells ${ }^{22}$-were elevated in the airway of $\mathrm{Axl}^{-1-}$ mice infected with influenza. Finally, airway macrophages from $\mathrm{Axl}^{-1-}$ mice displayed reduced uptake of apoptotic cells than those from wild-type mice (Figure 7l), indicating that Axl-mediated efferocytosis by airway macrophages is a critical step in the process of resolution of lung inflammatory disease upon viral infection.

\section{DISCUSSION}

We have found that under homeostatic conditions the TAM receptor Axl is preferentially expressed on murine airway macrophages and constitutively ligated by Gas6. Although constitutive expression of Axl has been reported on certain macrophage populations, such as splenic red pulp macrophages and Kupffer cells in the liver, ${ }^{5,17}$ we show that in the healthy lung, airway macrophages are the only population of immune cells expressing high levels of Axl. MerTK on the other hand, is expressed on all mature tissue macrophages. ${ }^{17}$ Also, despite constitutively binding Gas6, airway macrophages themselves expressed low levels of Gas6, and so may bind it in a paracrine manner after production from other cell types. Bone marrow, endothelial cells, and vascular smooth muscle cells express 


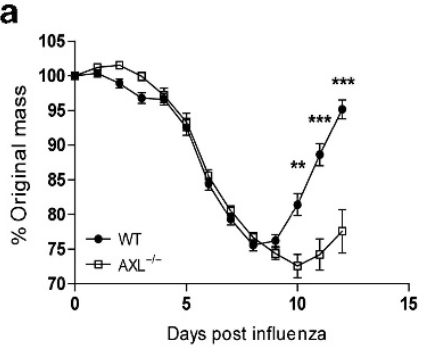

e
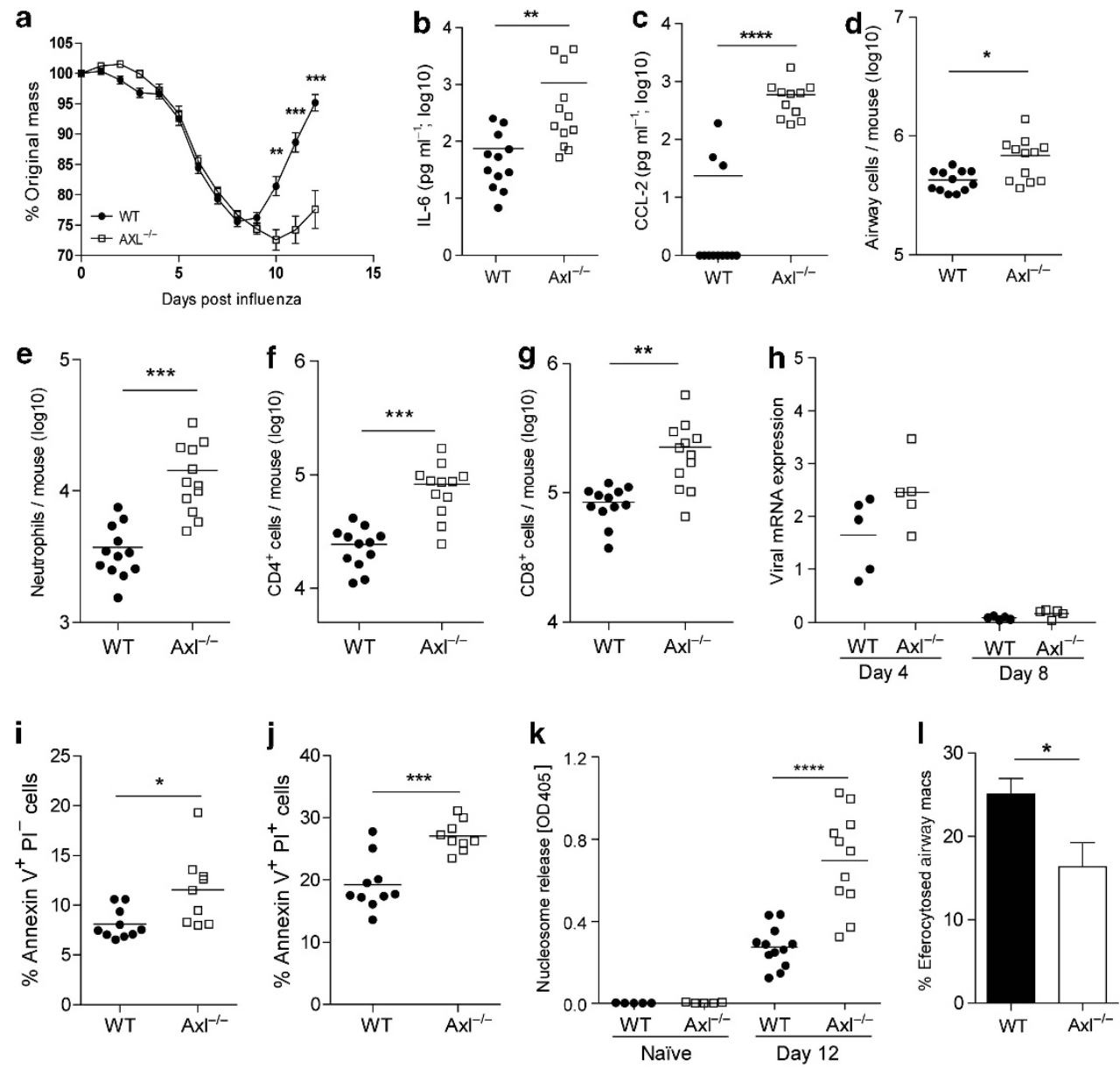

Figure 7 Increased severity of viral lung disease in influenza-infected $\mathrm{AxI}^{-1-}$ mice despite efficient clearance of viruses. (a) Change in body mass of wild-type (WT; closed symbol) and Axl ${ }^{-1-}$ (open symbol) mice infected with 7.5 p.f.u. influenza. Amount of interleukin (IL)-6 (b) and chemokine (C-C motif) ligand 2 (CCL-2) (c) in the bronchoairway lavage (BAL) fluid recovered on day 12 post influenza infection from WT and Axl ${ }^{-1-}$ mice. (d) Analysis of viable cells in the BAL from WT and $\mathrm{AxI}^{-1-}$ mice recovered on day 12 post influenza infection and counted using trypan blue exclusion. $(\mathbf{e}-\mathbf{g})$ Flow cytometric analysis of numbers of $(\mathbf{e})$ neutrophils, (f) CD4 ${ }^{+}$T cells, and $(\mathbf{g})$ CD8 ${ }^{+}{ }^{\top}$ cells in the BAL from WT and Axl ${ }^{-1-}$ mice on day 12 post influenza infection. (h) Influenza genomic mRNA copies recovered from the total lung of WT and Axl ${ }^{-1-}$ mice on days 4 and 8 post influenza infection. (i and j) Flow cytometric analysis of percentage of (i) early (Annexin $\mathrm{V}^{+}$propidium iodide ( $\left.\mathrm{PI}\right)^{-}$) and (j) late (Annexin $\mathrm{V}^{+} \mathrm{PI}^{+}$) apoptotic lymphocytes in the in the BAL from WT and $\mathrm{Axl}^{-1-}$ mice on day 10 post influenza infection. (k) Amount of nucleosomes released in the BAL fluid recovered from WT and $\mathrm{AxI}^{-1-}$ mice on day 0 (naïve) and day 12 post influenza infection. (I) Efficiency of uptake of apoptotic thymocytes by WT (filled bar) and AxI ${ }^{-1-}$ (open bar) airway macrophages measured by flow cytometry. (a-g and $\mathbf{k})$ are representative of two or three independent experiments with 9-12 mice per group. $(\mathbf{i}, \mathrm{j})$ Data from one experiment with 10 mice per group. $(\mathbf{h}, \mathrm{I})$ Representative of two independent experiments with four or five mice per group. ${ }^{\star} P<0.05$, ${ }^{* *} P<0.01,{ }^{* * *} P<0.001,{ }^{* * * *} P<0.0001$ vs. corresponding group; unpaired $t$-test.

Gas6 (refs 23-25) and we are the first to show differential expression of Gas6 in specific macrophage subsets, i.e., CD $11 b^{\text {hi }} \mathrm{CD} 11 c^{\text {intermediate }}$ monocyte/macrophages, but not $\mathrm{CD} 11 b^{\text {lo }} \mathrm{CD} 11 c^{\text {hi }}$ airway macrophages. This is likely to result in functional polarization of macrophages depending on their anatomical location. Although all airway macrophages also express a second TAM receptor, MerTK, Gas6 binding was lost in $\mathrm{Axl}^{-1-}$ macrophages, supporting the idea that $\mathrm{Axl}$ is the highest affinity receptor for this PtdSer-binding ligand, ${ }^{26}$ and suggesting a unique and non-redundant role of Axl and MerTK in regulating responses to apoptotic cells. In a recently proposed model, Axl has a dominant role in apoptotic cell uptake by macrophages under inflammatory conditions, whereas MerTK mediates macrophage responses to apoptotic cells in homeostasis and during immunosuppression. ${ }^{5}$ Consistently, we observed induction of Axl expression on macrophages by inflammatory stimuli in vitro and upon viral infection in vivo.

We have also found that GM-CSF induces Axl expression on peritoneal macrophages and BMDMs. GM-CSF is produced by a variety of cells, significantly airway epithelial type II cells, ${ }^{27}$ and is critical for airway macrophage development ${ }^{18}$ and the protection of airway epithelial integrity. ${ }^{19} \mathrm{GM}^{-\mathrm{CSF}^{-1}}{ }^{-1}$ mice lack airway macrophages ${ }^{19}$ and the presence of GM-CSF autoantibodies or mutations in the GM-CSF receptor $\alpha$ or $\beta$ chain leads to pulmonary airway proteinosis, ${ }^{28}$ a condition characterized by insufficient surfactant clearance by airway macrophages. Furthermore, GM-CSF-deficient mice 
experience severe morbidity to influenza virus infection, ${ }^{19}$ whereas treatment with GM-CSF increases airway macrophage numbers and protects against a lethal infection. ${ }^{29}$ Future studies will be needed to elucidate whether GM-CSF drives Axl expression in vivo and to determine which aspects of GM-CSF modulation of airway macrophage function depend on Axl activity.

Influenza infection of mice lacking Axl displayed a very similar proinflammatory phenotype to that reported for GM$\mathrm{CSF}^{-1-}$ animals. ${ }^{19} \mathrm{Axl}^{-1-}$ mice experienced excessive weight loss and a few reached the humane end point. Although in our experiments we used mice that lack Axl expression globally, recent evidence that airway macrophages are the main cell population responsible for efferocytosis in the respiratory tract supports the idea that the observed phenotype can be attributed to the lack of Axl on airway macrophages. ${ }^{19}$ Interestingly, viral clearance kinetics was unaffected, despite a number of viruses express PtdSer in their outer envelope ${ }^{30}$ and are opsonized by the TAM receptor ligands Gas6 or Protein S thereby acting as attachment factors enabling proximity to more specific entry receptors. ${ }^{31}$ Equivalent viral titers in $\mathrm{Axl}^{-1-}$ and wild-type mice in our experiments suggest that $\mathrm{Axl}$ is not important for control of influenza virus H1N1 infection, or may be compensated by other PtdSer-recognizing receptors. TIM1, e.g., promotes productive infection of a wide variety of enveloped viruses and increases their internalization, although for influenza $\mathrm{H} 7 \mathrm{~N} 1$ and SARS-CoV virions this does not result in a productive infection. ${ }^{32}$

Increased morbidity of influenza virus infection in $\mathrm{Axl}^{-1-}$ mice is more likely explained by the enhanced pulmonary inflammation and cytokine release, which may have arisen through two different processes. First, a lack of Axl signaling upon engagement of apoptotic cells may prevent induction of suppressor of cytokine signaling- 1 and -3 that inhibit cytokine and TLR signaling, ${ }^{2,33}$ and/or the nuclear factor- $\kappa \mathrm{B}$ repressor, Twist, that binds to the tumor necrosis factor promoter and inhibits nuclear factor- $\mathrm{\kappa B}-\mathrm{dependent}$ transcription. ${ }^{8}$ Similarly, the process of viral uptake has been shown to subvert antiviral immunity through a process termed "apoptotic mimicry", where viral envelope-expressed PtdSer sequesters Protein S and Gas6 that activate TAM receptors on bone marrow-derived dendritic cells causing a downregulation of TLR and IFN signaling pathways. ${ }^{34}$ An absence of Axl would remove any negative influence of PtdSerexpressing influenza virus on innate inflammation.

Second, failure to remove apoptotic cells leads to an increase in their membrane permeability and secondary necrosis that exposes otherwise hidden intracellular danger-associated molecular patterns, including uric acid, high-mobility group box 1, SAP 130, S100 proteins, nucleic acids in mitochondrial DNA, IL-1, and IL-33. ${ }^{35,36}$ These danger-associated molecular patterns are recognized by extracellular and intracellular pattern recognition receptors, such as the receptor for advanced glycation endproducts (RAGE) and the IL-33 receptor (ST2), ${ }^{37,38}$ that upon engagement produce a state of "sterile inflammation." ${ }^{36}$ Clearly timely removal of apoptotic cells is essential to prevent further inflammation and resolve inflammatory disease. The observed increase in nucleosome release in the airways of influenza-infected $\mathrm{Axl}^{-1-}$ mice in our study supports the idea of enhanced secondary necrosis, which could explain the observed elevated airway inflammatory cytokines. The airways of mice lacking GM-CSF (which induces Axl expression) following influenza infection are similarly enriched in dead cells and cellular debris indicating impaired clearance of apoptotic cells. ${ }^{19}$ In line with a pathogenic role of cellular components released from necrotic cells, circulating histones are direct mediators of lung inflammation and damage in patients with trauma-associated lung injury. ${ }^{39}$

Recently, a beneficial effect of administering an Axl-blocking antibody has been described during influenza virus infection, ${ }^{40}$ which is the opposite of what would be expected by blocking a receptor that activates anti-inflammatory pathways. The monoclonal antibody used in these studies prevents Gas6 binding to TAM receptors, causes receptor internalization, and inhibits downstream signaling. ${ }^{41}$ However, we and others ${ }^{5}$ show that Gas6 is constitutively bound to Axl-expressing airway macrophages; whether this antibody can disrupt this established interaction is not known. TAM receptors are pivotal inhibitory receptors that terminate cytokine receptor signaling. ${ }^{2,8,33}$ Chronic inflammation and systemic autoimmune disorders occur in TAM triple-knockout mice, ${ }^{33}$ phagocytes lacking TAM receptors display defective phagocytosis and overproduction of proinflammatory cytokines $\mathrm{s}^{4,7,42}$ and administration of protein $\mathrm{S}$ protects against LPS-induced lung injury. ${ }^{43}$ The beneficial effect of administering intraperitoneally an antibody that blocks Gas6 binding to Axl in virus-infected mice may in fact reflect antibody binding to soluble Axl that we have observed in the peripheral blood (mean concentration \pm s.e.m. $20.93 \pm 0.79 \mathrm{ng} \mathrm{ml}^{-1}, n=12$ ), which would reduce the competition for free Gas6 and promote membrane-bound Axl signaling. The distribution of administered antibody and whether it reached the high intensity of Axl expression on airway macrophages was not tested. ${ }^{40}$

Targeting Axl for the treatment of Gas6-Axl-related diseases is of high current interest. ${ }^{16} \mathrm{~A}$ small-molecule inhibitor that preferentially targets Axl inhibits breast cancer metastasis and angiogenesis, ${ }^{44}$ and is currently in phase 1 clinical trials (BGB324 by BergenBio). Complications, however, may arise from targeting a receptor that is also cleaved. With regards to the immune system, therapeutics sequestering the soluble form of Axl would be anti-inflammatory as it would remove competition for Gas6 by membrane tethered Axl. Conversely, blockade of Gas6 interaction with membrane-bound Axl or Axl enzymatic activity would be proinflammatory and lead to heightened inflammation due to secondary necrosis of apoptotic cells and a loss of anti-inflammatory signaling cascades. Current antibodies do not distinguish between soluble and membrane-bound forms of Axl at present and the drive to target this pathway stems from the known biology of membrane tethered Axl. The selective expression of Axl on airway, but not interstitial macrophages in the homeostatic state and its role in preventing secondary necrosis infers an important function that should not be ignored. 


\section{METHODS}

Detailed information on the experimental materials and methods used in this study can be found in Supplementary Material.

Mouse infection and sampling. Eight- to twelve- week-old female C57BL/6 (Harlan Olac, Bicester, UK) were maintained in specific pathogen-free conditions at Bio Safety Level 2, with a 12-h light/dark cycle, dry food pellets, and water ad libitum in accordance with the institutional and UK Home Office guidelines. The generation of $\mathrm{Axl}^{-1-}$ has been described previously, ${ }^{45}$ and all $\mathrm{Axl}^{-1-}$ mice used in this study have been back-crossed to C57BL/6J background for at least 10 generations. Mice were intranasally infected with 7.5 p.f.u. of influenza A virus, Puerto Rico/8/34(PR8), H1N1, and at specific time points euthanized by intraperitoneal injection of $3 \mathrm{mg}$ pentobarbitone and exsanguination via the femoral artery. Bronchoairway lavage, lung tissue, and samples from distal sites (peritoneum, small intestine, bone marrow, and blood) were harvested and dissociated to a single-cell suspension using methods described previously. ${ }^{20,46}$ Total macrophages from murine ears were extracted by splitting the dermis and epidermis in $0.8 \%$ trypsin for $30 \mathrm{~min}$ at $37^{\circ} \mathrm{C}$. Dermis and epidermis were minced with scissors and then the dermis fraction was further digested in $0.5 \mathrm{Wunch} \mathrm{U} \mathrm{ml}^{-1}$ of collagenase for $30 \mathrm{~min}$ at $37^{\circ} \mathrm{C}$. Both dermis and epidermis cell suspension were then passed through a $70-\mu \mathrm{m}$ sieve and combined. Red blood cells were lysed in whole blood and homogenized lung tissue using ammoniumchloride-potassium lysis buffer. Bronchoairway lavage fluid was retained for analysis of soluble Axl (R\&D systems, Abingdon, UK) or cytokines (eBioscience, Hatfield, UK) by ELISA. Viral titer was determined in lung homogenates by quantitative PCR on a QuantStudio 12K Flex PCR system (Life Technologies, Paisley, UK; fwd: 5'-GGACTGCAGCGTAGACGCTT-3'; rev: $5^{\prime}$-CATCCTGTTGTATATGAGGCCCAT- $\left.3^{\prime}\right)^{47}$ and quantified by the $\Delta \Delta C T$ method using QuantStudio $12 \mathrm{~K}$ Flex Software v1.1.1 (Life Technologies).

Flow cytometry. A total of $2 \times 10^{5}$ to $1 \times 10^{6}$ cells were incubated with near Infrared LIVE/DEAD fixable cell stain kit according to the manufacturer's instruction (Life Technologies) followed by anti-mouse CD16/32 Fc block (eBioscience) for $20 \mathrm{~min}$ at $4{ }^{\circ} \mathrm{C}$. After washing with PBA (phosphatebuffered saline containing $1 \%$ bovine serum albumin and $0.1 \%$ sodium azide), cells were stained with a customized extracellular antibody panel for $30 \mathrm{~min}$ at $4{ }^{\circ} \mathrm{C}$ in $\mathrm{PBA}$, and then fixed for 20 min with $2 \%$ paraformaldehyde or kept on ice with $5 \mu \mathrm{M}$ EDTA before sorting. Cells were run on a BD FACS Canto II collecting at least 10,000 events of the target population and analyzed using FlowJo (Tree Star, Ashland, OR). A BD Influx was used for sorting pure airway and peritoneal macrophages for quantitative PCR analysis. Alveolar macrophages were identified as $\mathrm{CD} 11 \mathrm{~b}^{\mathrm{lo}} \mathrm{CD} 11 \mathrm{c}^{\mathrm{hi}} \mathrm{F} 4 / 80^{\mathrm{hi}}$ and highly auto-fluorescent. Peritoneal macrophages were identified as $\mathrm{CD} 11 \mathrm{~b}^{\text {high }}$ $\mathrm{CD} 11 \mathrm{c}^{\mathrm{lo}} \mathrm{F} 4 / 80^{\mathrm{hi}}$. All lineage markers were purchased from eBioscience or BioLegend (San Diego, CA). Axl (clone 175128),
MerTK (polyclonal goat IgG, biotinylated), Tyro3 (clone 109646), and Gas6 (polyclonal goat IgG, biotinylated) antibodies were from R\&D Systems. Axl and Tyro3 antibodies were conjugated to Alexa Fluor-647 dye using a labeling kit (Life Technologies) according to the manufacturer's instruction.

Uptake of apoptotic thymocytes by macrophages. Protocol for measurement of apoptotic thymocyte uptake efficiency was adapted from ref. 48 and described in Supplementary Material.

Human alveolar macrophage isolation and culture. Alveolar macrophages were isolated as previously described ${ }^{49}$ and described in detail in Supplementary Material.

Statistics. GraphPad Prism version 5.04 (GraphPad Software, San Diego, California, USA) was used for all statistical calculations. For multiple data set analysis, analysis of variance with Bonferroni correction was applied. To compare two data sets, paired or unpaired $t$-tests were applied. Data are presented as the mean \pm standard error of the mean (s.e.m.). $P$ values $<0.05$ were considered significant.

SUPPLEMENTARY MATERIAL is linked to the online version of the paper at http://www.nature.com/mi

\section{ACKNOWLEDGMENTS}

We thank Ms Cecilia Forss and Mr Thomas Fenton (MCCIR, University of Manchester) for assistance with monocyte isolations, Dr Amy Saunders (MCCIR, University of Manchester) for extraction of cells from mouse ear skin, and Professor Greg Lemke and Dr Anna Zagorska (Salk Institute) for critical reading of the manuscript. This research was supported by Manchester Collaborative Centre for Inflammation Research, a joint initiative of the University of Manchester, AstraZeneca, and GlaxoSmithKline. T.J.B. was supported by the Wellcome Trust (reference 093612/Z/10/Z).

\section{DISCLOSURE}

The authors declare no conflict of interest.

c) 2015 Society for Mucosal Immunology

\section{REFERENCES}

1. Lemke, G. Biology of the TAM receptors. Cold. Spring. Harb. Perspect. Biol. 5, a009076 (2013).

2. Lemke, G. \& Rothlin, C.V. Immunobiology of the TAM receptors. Nat. Rev. Immunol. 8, 327-336 (2008).

3. Ravichandran, K.S. Beginnings of a good apoptotic meal: the find-me and eat-me signaling pathways. Immunity 35, 445-455 (2011).

4. Seitz, H.M., Camenisch, T.D., Lemke, G., Earp, H.S. \& Matsushima, G.K. Macrophages and dendritic cells use different Axl/Mertk/Tyro3 receptors in clearance of apoptotic cells. J. Immunol. 178, 5635-5642 (2007).

5. Zagorska, A., Traves, P.G., Lew, E.D., Dransfield, I. \& Lemke, G. Diversification of TAM receptor tyrosine kinase function. Nat. Immunol. 15, 920-928 (2014).

6. Alciato, F., Sainaghi, P.P., Sola, D., Castello, L. \& Avanzi, G.C. TNF-alpha, IL-6, and IL-1 expression is inhibited by GAS6 in monocytes/macrophages. J. Leukoc. Biol. 87, 869-875 (2010).

7. Rothlin, C.V. \& Lemke, G. TAM receptor signaling and autoimmune disease. Curr. Opin. Immunol. 22, 740-746 (2010).

8. Sharif, M.N. etal. Twist mediates suppression of inflammation by type I IFNs and Axl. J. Exp. Med. 203, 1891-1901 (2006).

9. Lu, Q. \& Lemke, G. Homeostatic regulation of the immune system by receptor tyrosine kinases of the Tyro 3 family. Science 293, 306-311 (2001).

10. Camenisch, T.D., Koller, B.H., Earp, H.S. \& Matsushima, G.K. A novel receptor tyrosine kinase, Mer, inhibits TNF-alpha production and 
lipopolysaccharide-induced endotoxic shock. J. Immunol. 162, 34983503 (1999).

11. Deng, T., Zhang, Y., Chen, Q., Yan, K. \& Han, D. Toll-like receptor-mediated inhibition of Gas6 and ProS expression facilitates inflammatory cytokine production in mouse macrophages. Immunology 135, $40-50$ (2012).

12. Binder, M.D. \& Kilpatrick, T.J. TAM receptor signalling and demyelination. Neuro-Signals 17, 277-287 (2009).

13. Ekman, C., Linder, A., Akesson, P. \& Dahlback, B. Plasma concentrations of Gas6 (growth arrest specific protein 6) and its soluble tyrosine kinase receptor sAxl in sepsis and systemic inflammatory response syndromes. Crit. Care 14, R158 (2010).

14. Guignant, C. et al. Increased MerTK expression in circulating innate immune cells of patients with septic shock. Intensive Care Med. 39, 1556-1564 (2013).

15. Hussell, T. \& Bell, T.J. Alveolar macrophages: plasticity in a tissue-specific context. Nat. Rev. Immunol. 14, 81-93 (2014).

16. Verma, A., Warner, S.L., Vankayalapati, H., Bearss, D.J. \& Sharma, S. Targeting Axl and Mer kinases in cancer. Mol. Cancer Ther. 10, 1763-1773 (2011).

17. Gautier, E.L. et al. Gene-expression profiles and transcriptional regulatory pathways that underlie the identity and diversity of mouse tissue macrophages. Nat. Immunol. 13, 1118-1128 (2012).

18. Guilliams, M. et al. Alveolar macrophages develop from fetal monocytes that differentiate into long-lived cells in the first week of life via GM-CSF. J. Exp. Med. 210, 1977-1992 (2013).

19. Schneider, C. et al. Alveolar macrophages are essential for protection from respiratory failure and associated morbidity following influenza virus infection. PLoS Pathog. 10, e1004053 (2014).

20. Snelgrove, R.J. et al. A critical function for CD200 in lung immune homeostasis and the severity of influenza infection. Nat. Immunol. 9, 1074-1083 (2008).

21. Didierlaurent, A. et al. Sustained desensitization to bacterial Toll-like receptor ligands after resolution of respiratory influenza infection. J. Exp. Med. 205, 323-329 (2008).

22. Holdenrieder, S. \& Stieber, P. Clinical use of circulating nucleosomes. Crit. Rev. Clin. Lab. Sci. 46, 1-24 (2009).

23. Avanzi, G.C. et al. GAS6, the ligand of Axl and Rse receptors, is expressed in hematopoietic tissue but lacks mitogenic activity. Exp. Hematol. 25, 1219-1226 (1997).

24. Manfioletti, G., Brancolini, C., Avanzi, G. \& Schneider, C. The protein encoded by a growth arrest-specific gene (Gas6) is a new member of the vitamin-K-dependent proteins related to protein-S, a negative coregulator in the blood-coagulation cascade. Mol. Cell. Biol. 13, 4976-4985 (1993).

25. Nakano, T. et al. Vascular smooth muscle cell-derived, Gla-containing growth-potentiating factor for Ca-mobilizing growth factors. J. Biol. Chem. 270, 5702-5705 (1995).

26. Nagata, K. et al. Identification of the product of growth arrest-specific gene 6 as a common ligand for Axl, Sky, and Mer receptor tyrosine kinases. J. Biol. Chem. 271, 30022-30027 (1996).

27. Trapnell, B.C. \& Whitsett, J.A. Gm-CSF regulates pulmonary surfactant homeostasis and alveolar macrophage-mediated innate host defense. Annu. Rev. Physiol. 64, 775-802 (2002).

28. Kitamura, T. et al. Idiopathic pulmonary alveolar proteinosis as an autoimmune disease with neutralizing antibody against granulocyte/ macrophage colony-stimulating factor. J. Exp. Med. 190, 875-880 (1999).
29. Huang, F.F. et al. GM-CSF in the lung protects against lethal influenza infection. Am. J. Respir. Crit. Care. Med. 184, 259-268 (2011).

30. Mercer, J. Viral apoptotic mimicry party: P.S. Bring your own Gas6. Cell Host Microbe 9, 255-257 (2011).

31. Morizono, K. et al. The soluble serum protein Gas6 bridges virion envelope phosphatidylserine to the TAM receptor tyrosine kinase Axl to mediate viral entry. Cell Host Microbe 9, 286-298 (2011).

32. Jemielity, S. et al. TIM-family proteins promote infection of multiple enveloped viruses through virion-associated phosphatidylserine. PLOS Pathog. 9, e1003232 (2013).

33. Rothlin, C.V., Ghosh, S., Zuniga, E.I., Oldstone, M.B. \& Lemke, G. TAM receptors are pleiotropic inhibitors of the innate immune response. Cell 131, 1124-1136 (2007).

34. Bhattacharyya, S. et al. Enveloped viruses disable innate immune responses in dendritic cells by direct activation of TAM receptors. Cell Host Microbe 14, 136-147 (2013).

35. Rock, K.L., Lai, J.J. \& Kono, H. Innate and adaptive immune responses to cell death. Immunol. Rev. 243, 191-205 (2011).

36. Poon, I.K., Lucas, C.D., Rossi, A.G. \& Ravichandran, K.S. Apoptotic cell clearance: basic biology and therapeutic potential. Nat. Rev. Immunol. 14, 166-180 (2014).

37. Kono, H. \& Rock, K.L. How dying cells alert the immune system to danger. Nat. Rev. Immunol. 8, 279-289 (2008).

38. Bonilla, W.V. et al. The alarmin interleukin-33 drives protective antiviral CD8(+) T cell responses. Science 335, 984-989 (2012).

39. Abrams, S.T. et al. Circulating histones are mediators of trauma-associated lung injury. Am. J. Respir. Crit. Care Med. 187, 160-169 (2013).

40. Shibata, T. et al. Axl receptor blockade ameliorates pulmonary pathology resulting from primary viral infection and viral exacerbation of asthma. J. Immunol. 192, 3569-3581 (2014).

41. Ye, X. et al. An anti-Axl monoclonal antibody attenuates xenograft tumor growth and enhances the effect of multiple anticancer therapies. Oncogene 29, 5254-5264 (2010).

42. Cohen, P.L. et al. Delayed apoptotic cell clearance and lupus-like autoimmunity in mice lacking the c-mer membrane tyrosine kinase. J. Exp. Med. 196, 135-140 (2002).

43. Takagi, T. et al. Direct effects of protein $S$ in ameliorating acute lung injury. J. Thromb. Haemost. 7, 2053-2063 (2009).

44. Holland, S.J. et al. R428, a selective small molecule inhibitor of Axl kinase, blocks tumor spread and prolongs survival in models of metastatic breast cancer. Cancer Res. 70, 1544-1554 (2010).

45. Lu, Q. et al. Tyro-3 family receptors are essential regulators of mammalian spermatogenesis. Nature 398, 723-728 (1999).

46. Wirtz, S., Neufert, C., Weigmann, B. \& Neurath, M.F. Chemically induced mouse models of intestinal inflammation. Nat. Protoc. 2, 541-546 (2007).

47. van Elden, L.J., Nijhuis, M., Schipper, P., Schuurman, R. \& van Loon, A.M. Simultaneous detection of influenza viruses $A$ and $B$ using real-time quantitative PCR. J. Clin. Microbiol. 39, 196-200 (2001).

48. Miksa, M., Komura, H., Wu, R., Shah, K.G. \& Wang, P. A novel method to determine the engulfment of apoptotic cells by macrophages using pHrodo succinimidyl ester. J. Immunol. Methods 342, 71-77 (2009).

49. Kent, L.M., Fox, S.M., Farrow, S.N. \& Singh, D. The effects of dexamethasone on cigarette smoke induced gene expression changes in COPD macrophages. Int. Immunopharmacol. 10, 57-64 (2010). 